\title{
PRINCIPIOS DE CONTABILIDAD GENERALMENTE ACEPTADOS \\ Vigencia y Aplicación
}

CPC.JORGE J.GAVELÁNIZAGUIRRE (*)

\section{INTRODUCCION}

Si la elaboración de la información contable estuviera supeditado únicamente a la decisión del Contador, se obtendría información formulado con criterios diferentes. La contabilidad durante el transcurso del tiempo, ha elaborado reglas que sirven de guías para la registración contable y que en convenciones de contabilidad se han aceptado como útiles y necesarios para uniformizar el criterio contable. Éstas han evolucionado en concordancia con los cambios y necesidades sociales, adaptándose a la economía y a las empresas en particular. Estas reglas se denominan "principios". En términos corrientes, es la norma o idea fundamental que rige el pensamiento o la conducta.

Eric L. Kohler en su obra "Diccionario para Contador" define a los Principios de Contabilidad como "Cuerpo de doctrina asociado con la contabilidad que sirve de explicación de las actividades corrientes o actuales y como guía en la selección de convencionalismos o procedimientos".

Los PCGA (Principios de Contabilidad Generalmente Aceptados) son un conjunto de reglas generales y normas que sirven de guía contable para formular criterios referidos a la medición del patrimonio y a la información de los elementos patri- moniales y económicos de un ente. Los PCGA constituyen parámetros para que la confección de los estados financieros sea sobre la base de métodos uniformes de técnica contable.

\section{¿POR QUÉ A LAS REGLAS CONTABLES SE LES DENOMINA PCGA?}

Precisando la definición de cada vocablo, se tiene:

Principio.- Cualquiera de las máximas o normas por las que cada uno se guía. Regla general adoptada para servir como guía de una acción.

General.- Común, usual, frecuente, prevalecente. admitir.

Aceptar,- Aprobar, dar por bueno,

En consecuencia, los PCGA constituyen reglas generales adoptadas como guías y fundamentos de aplicaciones contables, aprobados como buenos y prevalecentes.

\section{¿QUÉ EFECTOS O CUALIDADES TIENEN LOS PCGA?}

- Han de ser razonables y prácticos en su aplicación. 
- Han de producir resultados equitativos y comprensibles.

- Han de ser aplicables bajo circunstancias variables.

- Han de ser susceptibles de observarse uniformemente.

- Han de producir resultados comparables de periodo a periodo y entre compañías.

- Han de ajustarse a los resultados generalmente aceptados que sustentan los principios de contabilidad en general.

El Plan Contable General Revisado reconoce que los principios que se exponen a continuación, son los fundamentales y básicos para el adecuado cumplimiento de los fines de la contabilidad.

1. Equidad

2. Partida doble

3. Ente

4. Bienes económicos

5. Moneda común denominador

6. Empresa en marcha

7. Valuación al costo

8. Período

9. Devengado

10. Objetividad

11. Realización

12. Prudencia

13. Uniformidad

14. Significación o importancia relativa

15: Exposición

Los PCGA señalados con la excepción de Partida Doble son los que fueron aprobados en la VII Conferencia Interamericana de Contabilidad realizada en Mar de Plata, Argentina, en el año 1965, habiendo algunos cambiado de denominación, pero siguen siendo los mismos. Así en la versión original se denomina Moneda de cuenta,
Ejercicio y Materialidad, en vez de Moneda común Denominador, Período y Significación o Importancia relativa, respectivamente. Estos cambios y la incorporación de la Partida Doble como principio fue establecido en el Perú a través del Plan Contable General del año 1973, luego modificado y aprobado con Resolución de CONASEV $\mathrm{N}^{\circ}$ 006-84-EFC/94.10 del 15/02/84 de vigencia actual. La Partida Doble no es un PCGA, ésta es únicamente una técnica de registro o medio de instrumentación contable, algo equivalente (guardando la distancia del caso) a la Partida Simple de vigencia durante siglos y sustituida años después de aparecer la obra "Summad de Arithmetica,Geometria, Proportioni et proportionalita" del Padre de la Partida Doble Fray Luca Pacioli, impresa en su primera edición en Venecia en el año 1494. En la parte del texto titulada "Tractatus de Com-putis et Scripturis" (Tratado de las Cuentas y de la Escritura) es donde expone la forma de llevar las cuentas por Partida Doble.

No obstante el tiempo transcurrido y múltiples revisiones efectuadas, los PCGA siguen vigentes, habiéndose ampliado y precisado su aplicación a fines o situaciones específicas a través de las Normas Internacionales de Contabilidad (NIC). Ambos son complementarios, es como si fueran Ley y Reglamento respectivamente, siendo imprescindible para el profesional contable tener suficiente dominio de éstos, ya que son el soporte de su conocimiento aplicativo; en caso extremo, podría "perdonarse" su insuficiente conocimiento de los NIC's, pero no de los PCGA, que es lo que constituye el objeto del presente trabajo y que se expone a continuación: 


\section{EQUIDAD}

Enunciado textual según el PCGR:

Es el principio fundamental que debe orientar la acción del profesional contable en todo momento y se anuncia así:

La equidad entre intereses opuestos debe ser una preocupación constante en contabilidad, puesto que los que se sirven de, o utilizan los datos contables pueden encontrarse ante el hecho de que los intereses particulares se hallen en conflicto. De esto se desprende que los estados financieros deben prepararse de tal modo que reflejen, con equidad, los distintos intereses en juego en una empresa dada.

\section{Comentario:}

El principio de equidad es sinónimo de imparcialidad y justicia, y tiene la condición de postulado básico. Es una guía de orientación, en íntima relación con el sentido de lo ético y justo, para la evaluación contable de los hechos que constituyen el objeto de la contabilidad, y se refiere a que la información contable debe prepararse con equidad respecto a terceros y a la propia empresa, a efecto de que los estados financieros reflejen equitativamente los intereses de las partes y que la información que brindan sea lo más justa posible para los usuarios interesados, sin favorecer o desfavorecer a nadie en particular. Bajo el principio de equidad se debe compatibilizar intereses opuestos.

Si el sentido de justicia entendido como "virtud que se inclina a dar a cada uno lo que le pertenece" se aplica a cada ejercicio, se debe asignar a este su correspondiente ingreso, costo y gasto.

Si por ejemplo, se ha recibido la factura \# 0290 del $21 / 10 / 2000$ por $S / .45 .000$, más IGV S/. 8.100, total S/. 53.100, para realizar una campaña publicitaria por el periodo
01/11/2000 al 31/01/2001, el que según los estudios efectuados ha de tener efecto en el incremento de las ventas a partir del 01/12/ 2000 , lo que ha sido constatado en los hechos. ¿Por cuál suma y a partir de que fecha se debe aplicar los resultados? Respuesta: En el mes de diciembre 2000 se aplica a gastos del periodo $\mathrm{S} / \mathrm{15.000}$ y se difiere $S / .30 .000$ para el siguiente año.

\section{PARTIDA DOBLE}

Enunciado textual, según el PCGR:

Los hechos económicos y jurídicos de la empresa se expresan en forma cabal aplicando sistemas contables que registran los dos aspectos de cada acontecimiento, cambios en el activo y en el pasivo (participaciones) que dan lugar a la ecuación contable.

\section{Comentario:}

El principio de la partida Doble o dualidad es la base del método contable, se le define como: "A toda partida registrada en el Debe le corresponde otra partida registrada en el Haber" o "No hay deudor sin acreedor, ni acreedor sin deudor". Esta duplicidad presenta siempre una igualdad entre las sumas que figuran en el Debe y el Haber, lo que permite la comprobación de la igualdad de las registraciones. Mediante la partida Doble se registra los cambios en el activo (aplicación de fondos) y en el pasivo (origen de fondos) y capital. El activo es cualquier derecho o bien que se posee y que tienen un valor monetario. El pasivo es la suma que se adeuda a terceros, es el derecho de los acreedores, excluyendo al propietario(s) del negocio. La suma que este último ha invertido se denomina capital.

Los tres elementos mencionados están unidos por una relación fundamental, producto de la suma de transacciones registradas sobre la base de la partida doble 
denominada ecuación contable, la que expresa el equilibrio entre las partes, como:

ACTIVO $=$ PASIVO + CAPITAL

En la partida doble cada transacción tiene un elemento de débito (o cargo) y otro de crédito (o abono) por igual suma monetaria, toda operación que registra la contabilidad afecta por lo menos a dos partes.

\section{ENTE}

\section{Enunciado textual, según el PCGR:}

Los estados financieros se refieren siempre a un ente, donde el elemento subjetivo o propietario es considerado como tercero. El concepto de ente es distinto del de persona, ya que una misma persona puede producir estados financieros de varios entes de su propiedad.

\section{Comentario:}

El principio de ente o Principio de Entidad establece el supuesto de que el patrimonio de la empresa se independiza del patrimonio personal del propietario, considerado como un tercero. Se efectúa una separación entre la propiedad (accionistas o socios o propietario) y la administración (gerencia) como procedimiento indispensable de rendir cuenta por estos últimos. El ente tiene vida propia $y$ es sujeto de derechos y obligaciones, distinto de las personas que lo formaron.

Los propietarios son acreedores de las empresas que han formado y aunque tengan varias empresas, cada una se trata como una entidad separada, por lo que el propietario es un acreedor más de la entidad, al que contablemente se le representa con la cuenta 50 Capital.
Es frecuente observar en las empresas unipersonales o sociedades de propiedad mayoritaria de una persona, la mala práctica de utilizar los recursos monetarios o bienes de la empresa en beneficio o uso personal, cancelándose facturas o asumiendo gastos particulares ajenos a la empresa, situación que debe obligar a adoptar normas adecuadas de registración contable que diferencien los bienes, derechos u obligaciones personales, de los que corresponden a la empresa. Contablemente se debe distinguir entre la entidad y los intereses ajenos al mismo.

Si por ejemplo, la empresa Miraflores E.I.R.L. dedicada a la compra-venta de textiles, propiedad de Lizardo Alzamora y Mayo, ha recibido las siguientes boletas de venta: $\mathrm{N}^{\circ} 1199$ del 02/03/2000 del colegio Particular "Santa Catalina", por S/.1.500, por matrícula escolar del hijo del propietario. - $\mathrm{N}^{\circ} 0127$ del 14/03/2000 de Comercial Lurin S.A.C. por S/. 900, por compra de una cocina de uso doméstico INRESA y un balón de gas.

¿Debe ser registrado en los libros de contabilidad los documentos señalados? Ambos documentos están referidos a gastos personales del propietario y bienes ajenos a la empresa, por lo que debe aplicarse el siguiente tratamiento:

Si los documentos están pendientes de pago, no registrarlos en libros; si en cambio se han pagado con fondos de la empresa, registarlos en libros, pero no a cuentas de gastos y de activo fijo, sino a cuentas por cobrar diversas por S/. 2.400 a efectos de gestionar el reembolso o recuperación correspondiente.

De acuerdo a lo enunciado, separar las transacciones de la entidad de las del propietario(s) es un objetivo importante de la contabilidad. Así lograremos obtener 
información correcta de la administración de los recursos provenientes del propietario(s), bancos, y proveedores confiados a los directivos de la entidad y mediremos el desempeño de sus funciones.

\section{BIENES ECONÓMICOS}

\section{Enunciado textual, según el PCGR:}

Los estados financieros se refieren siempre a bienes económicos; es decir, bienes materiales e inmateriales que poseen valor económico y por ende, susceptibles de ser valuados en términos monetarios.

\section{Comentario:}

Se registra en libros todo acto o bien susceptible de valor de intercambio, independientemente de la forma cómo se ha obtenido, y por el cual alguien está dispuesto a pagar un precio.

Cualquier activo, como caja, mercaderías, activos fijos en poder y/o uso de la entidad y sobre el cual se ejerce derecho, sin estar acreditado necesariamente la propiedad de la misma, mientras no entre en conflicto con terceros que también reclaman la propiedad, son susceptibles de registrar en libros en vía de regularización, a través de un asiento de ajuste, tratamiento que se hace extensivo a las diferencias en los costos de adquisición o registro en fecha anterior.

Caso típico de la aplicación de este PCGA es el proveniente de los inventarios físicos de existencias, en que se encuentra unidades que no están registrados en libros, y siempre que no sean de terceros, se procede a aperturar su tarjeta (kardex) de control físico y a formular el asiento contable de regularización.

\section{MONEDA COMÚN DENOMINADOR}

Enunciado textual, según PCGR:

Los estados financieros reflejan el patrimonio mediante un recurso que se emplea para reducir todos sus componentes heterogéneos a una expresión, que permita agruparlos y compararlos fácilmente. Este recurso consiste en elegir una moneda y valorizar los elementos patrimoniales aplicando un precio a cada unidad.

Generalmente, se utiliza como denominador común la moneda que tiene curso legal en el país en que funciona el ente. En el Perú, de conformidad con dispositivos legales, la contabilidad se lleva en moneda nacional.

\section{Comentario:}

La elaboración de estados financieros basados en la premisa de que la moneda es una unidad. Todos los países, unos más que otros, son víctimas de la inflación, por lo cual en el transcurso del tiempo se "mezclan" monedas de diferente poder adquisitivo. Esta mezcla se da, especialmente, en el caso de los activos fijos. Por ejemplo, si en enero de 1998 se compró un terreno, tres años después se vuelve a comprar otro en el mismo lugar y en iguales condiciones, el precio de adquisición es posible que sea mayor, debido principalmente a la pérdida de poder adquisitivo de la moneda, por lo que las transacciones del último periodo no se puede mezclar con los de períodos anteriores, a costa de obtener por resultado la combinación de unidades monetarias de diferente poder adquisitivo, lo que incidiría en la distorsión de los estados financieros.

En el caso de establecer el costo de fabricación del período con la participación de sus tres elementos: materia prima, mano de obra y gastos de fábrica, cada uno de ellos estará afectado en diferentes niveles por 
la inflación; incluso algunos podrían no haber sido afectados durante un tiempo, como es el caso de las remuneraciones. La materia prima utilizada en la elaboración podría haber sido asumida al valor del momento (supuesto que se compre para uso inmediato), luego su costo ya está afectado por la inflación, al pagar o comprometer su pago a un precio mayor con respecto a compra anterior. En el caso de los Gastos de Fábrica, será una mezcla de valores no afectados por la inflación durante un tiempo, como es por ejemplo el caso de la depreciación y del alquiler y pólizas de seguro contratados en moneda nacional; en otros casos si tendrá un efecto inmediato, como es el empleo de combustibles y energía eléctrica.

No obstante lo expresado en el párrafo precedente, la moneda es el único denominador de uso práctico para hacer homogéneo el registro de operaciones diferentes, sean éstos referidos a adquisición de activos o gastos incurridos en fechas y periodos diferentes. La fluctuación de la moneda en el tiempo por pérdida de su poder adquisitivo no altera la validez de los PCGA, es factible su corrección mediante factores de actualización monetaria, a efectos de lograr aproximarse a una objetividad más consistente en la presentación de los estados financieros.

\section{EMPRESA EN MARCHA}

\section{Enunciado textual, según el PCGR:}

Salvo indicación expresa en contrario, se entiende que los estados financieros pertenecen a una "empresa en marcha", considerándose que el concepto que informa la mencionada expresión, se refiere a todo organismo económico cuya existencia temporal tiene plena vigencia y proyección.

\section{Comentario:}

El PCGA "empresa en marcha", también conocido como " Continuidad de la empresa" se basa en la presunción de que la empresa continuará sus operaciones por un tiempo indefinido y no será liquidado en un futuro previsible, salvo que existan situaciones como: significativas y contínuas pérdidas, insolvencia, etc. En consecuencia, la valuación contable de los hechos serán los mismos u homogéneos, mientras no cambie su situación o cese en sus actividades, en cuyo caso se aplicará criterios de realización o de mercado. En caso de venta o liquidación de la empresa, recién cambiaría el tratamiento de registro de las operaciones, ésta se orientaría a medir el valor actual que tiene la empresa para el comprador la continuidad de la empresa en el tiempo justifica que los activos fijos sean registrados a su costo de adquisición, despreciándolos sobre estos valores, cargando a gastos en los ejercicios en que presta servicios, sin tomar en cuenta su valor en el mercado (valor de realización), porque el bien está en uso y no hay necesidad inmediata de venderlo, por consiguiente la fluctuación de precios en el mercado respecto al bien adquirido, no causa pérdida ni ganancia.

Una empresa en marcha agrega valor a los recursos que usa, estableciendo su ganancia por diferencia entre el valor de venta y el costo de los recursos utilizados para generar los ingresos, mostrando en el Balance General los recursos no consumidos a su costo de adquisición, y no a su valor actual de mercado.

\section{VALUACIÓN AL COSTO}

Enunciado textual, según el PCGR:

El valor de costo -adquisición o producción-constituye el criterio principal 
y básico de valuación, que condiciona la formulación de los estados financieros llamados de situación, en correspondencia también con el concepto de "empresa en marcha", razón por la cual esta norma adquiere el carácter de principio.

Esta afirmación no significa desconocer la existencia y procedencia de otras reglas y criterios aplicables en determinadas circunstancias, sino que, por el contrario, significa afirmar que en caso de no existir una circunstancia especial que justifique la aplicación de otro criterio, debe prevalecer el costoadquisición o producción-como concepto básico de valuación.

Por otra parte, las fluctuaciones de valor de la moneda común denominador, con su secuela de correctivos que inciden o modifican las cifras monetarias de los costos de determinados bienes, no constituye, asimismo, alteraciones al principio expresado, sino que, en sustancia, representen simples ajustes a la expresión numérica de los respectivos costos.

\section{Comentario:}

Cuando los activos se registran al precio pagado para adquirirlo, se está haciendo prevalecer el costo como concepto básico de valuación, cuyo concepto se relaciona con el principio de "empresa en marcha" o "continuidad de la empresa" en que el costo es el efecto de la causa. El principio de costo condiciona la valuación de los bienes al concepto de "erogación efectivamente producida" o costos comprometidos y necesarios para la incorporación al patrimonio.

Si bien el valor real de un bien varía con el transcurso del tiempo, la contabilidad no refleja el valor actual de los activos, con respecto a la fecha de compra; si por ejemplo un edificio adquirido en $S / .50 .000$ pudiera dos años después estar vendiéndose en
S/.60.000 o en S/. 40.000, no se registrará en la contabilidad ningún cambio, salvo que, como en el caso del Perú, se aplicara el ajuste por inflación al cierre del ejercicio de acuerdo a la normatividad establecida. En libros no se muestra el posible valor de venta de los bienes.

La valuación al costo es también aplicable a los activos inmateriales como los intangibles, que se registran a la suma efectivamente pagada por su adquisición, aunque se pueda determinar que con el transcurso del tiempo o por alguna circunstancia se estime que su valor sea mayor al de su adquisición. El registro a base de valores estimados de mercado generaría cierta inseguridad, con respecto a que si la valorización corresponde a valores justos o razonables.

El PCGA de "valuación al costo" implica que no debe adoptarse como criterio de valuación el de "valor de mercado", entendiéndose como tal el "costo de reposición o refrabricación". Sin embargo, el criterio de "valuación al costo" ligado al de "empresa en marcha", cuando esta última condición se interrumpe o desaparece, por estar la empresa en liquidación, incluso fusión, el criterio aplicable será el de "valor de mercado" o "valor de probable realización", según corresponda.

\section{PERIODO}

\section{Enunciado textual, según el PCGR:}

En la "empresa en marcha" es indispensable medir el resultado de la gestión de tiempo en tiempo, ya sea para satisfacer razones de administración, legales, fiscales o para cumplir con compromisos financieros.

El lapso que media entre una fecha y otra se llama periodo. Para los efectos del Plan Contable General, este periodo es de doce meses y recibe el nombre de Ejercicio. 


\section{Comentario:}

El PCGA de "ejercicio" (periodo) significa dividir la marcha de la empresa en periodos uniformes de tiempo a efectos de medir los resultados de la gestión y establecer la situación financiera del ente y cumplir con las disposiciones legales y fiscales establecidas, particularmente para determinar el Impuesto a la Renta y la distribución del resultado. En esta información periódica también están interesados terceras personas, como es el caso de las entidades bancarias y potenciales inversionistas.

Las empresas tienen una duración indefinida e ilimitada; por consiguiente, sus resultados sólo se conocen sino hasta que concluya su existencia, por lo que es necesario dividir el desarrollo de sus actividades en periodos contables y establecer al cierre del periodo los resultados de operación y su situación financiera e informar de los hechos importantes que han generado cambios en la participación de los propietarios de la empresa durante ese lapso de tiempo.

\section{DEVENGADO}

Enunciado textual, según el PCGR:

Las variaciones patrimoniales que se deben considerar para establecer el resultado económico, son los que corresponden a un ejercicio sin entrar a distinguir si se han cobrado o pagado durante dicho periodo.

\section{Comentario:}

En la aplicación del principio de "devengado" se registran los ingresos o gastos en el periodo contable al que se refiere, a pesar de que el documento sustentatorio tuviera fecha del siguiente ejercicio o que el desembolso pueda ser hecho todo o en parte en el ejercicio siguiente. Este principio elimina la posibilidad de aplicar el criterio de la "percibido" para la atribución de resultados. Este último método se halla al margen de los PCGA.

Los servicios o bienes utilizados o consumidos en el ejercicio, aunque no hayan sido cancelados, ni siquiera se conozca exactamente el monto a pagar, obliga a su registro formulando un asiento de ajuste, lo que podría a su vez implicar que se afecte a gastos del ejercicio o del siguiente.

Devengar significa reconocer y registrar en cuentas a determinada fecha eventos o transacciones contabilizables, como intereses por cobrar sobre un préstamo concedido, remuneraciones vencidas pendientes de pago, regalías por remesar, depreciación de activos frjos, etc. El término devengado se aplica sobre todo a los servicios más que a los activos adquiridos.

\section{OBJETIVIDAD}

Enunciado textual, según el PCGR:

Los cambios en el activo, pasivo y en la expresión contable del patrimonio neto, se deben reconocer formalmente en los registros contables, tan pronto como sea posible medirlos objetivamente y expresar esta medida en términos monetarios.

\section{Comentario:}

La objetividad consiste en evaluar contablemente los hechos y actividades económicas y financieras en que participa la empresa, tal como éstos se presentan, libre de prejuicios. Los estados financieros deberán reflejar un punto de vista razonable y neutral de las actividades desarrolladas, y ser susceptibles de verificación por terceros.

Objetividad en términos contables es una "evidencia" que respalda el registro de 
la variación patrimonial. La evidencia puede estar constituida por documentos mercantiles convencionales o por hechos no necesariamente documentados, caracterizado por cierto grado de certidumbre, como es la variación del tipo de cambio de una moneda extranjera de una fecha a otra, variación en la cotización de títulos como acciones y bonos, estimación de la probable incobrabilidad de letras por cobrar, provisión por mercaderías obsoletas, etc.

\section{REALIZACIÓN}

\section{Enunciado textual, según el PCGR:}

Los resultados económicos se registran cuando sean realizados, o sea cuando la operación que los origina queda perfeccionada desde el punto de vista de la legislación o de las prácticas comerciales aplicables y se hayan ponderado fundamentalmente todos los riesgos inherentes a tal operación. Se establecerá como carácter general que el concepto "realizado" participa del concepto de "devengado".

\section{Comentario:}

Con respecto a las operaciones de compra-venta, se considera vendido un bien cuando se concreta la entrega de la mercadería o es puesta a disposición del comprador, lo que permite atribuir objetivamente su correspondiente costo. En caso de venta de servicios formalizados por contratos, en los cuales se cobra por anticipado, los resultados serán reconocidos en función de la realización periódica de los servicios, lo que implica afectar a resultados, generalmente en forma mensual.

En las operaciones de compra-venta a largo plazo, se admite la atribución a resultados en función de las cuotas cobradas o puestas a disposición, lo que constituye una excepción al principio de "devengado".
Las operaciones, en las que la atribución a los resultados dependen del tiempo, los gastos e ingresos se mantendrán como activos y pasivos respectivamente, hasta que el tiempo determine su conversión en resultados, como por ejemplo, el servicio de alquiler, seguro, etc.

En aplicación del principio de "realización" las transacciones internas que no modifican la estructura de los recursos (activos) o sus fuentes (pasivos), como es la determinación del costo de fabricación constituido por la suma de la materia prima, mano de obra y gastos de fábrica aplicados, se procede a registrar contablemente, al evidenciarse la utilización de los recursos indicados para obtener un producto elaborado.

Los ajustes por inflación, así como aquellos efectuados para empalmar los ingresos de un ejercicio contable con los gastos incurridos para su obtención (principio de enfrentamiento o correspondencia), se efectúan bajo los alcances del PCGA de realización.

\section{PRUDENCIA}

Enunciado textual, según el PCGR:

Significa que cuando se deba elegir entre dos valores para un elemento del activo, normalmente, se debe optar por el más bajo, o bien que una operación se contabilice de tal modo, que la participación del propietario sea menor.

Este principio general se puede expresar también diciendo: "contabilizar todas las pérdidas cuando se conocen y las ganancias solamente cuando se hayan realizado".

La exageración en la aplicación de este principio no es conveniente si resulta en detrimento de la presentación razonable de la 
situación financiera y del resultado de las operaciones.

\section{Comentario:}

Lo enunciado implica que en caso que el contador tuviera dos o más opciones contables a aplicar, tiene que optar por el registro de aquella que muestre en libros un menor valor del activo o que incida en una menor utilidad de la empresa.

El principio de Prudencia (conservador) no sólo está referido a transacciones con terceros, sino también a situaciones internas de la empresa que se derivan de cambios en el tiempo del valor de los activos y pasivos, determinándose por comparación entre una situación anterior a otra posterior, variaciones que incrementan odisminuyen su valor, por consiguiente las ganancias o pérdidas que se deriven de la comparación deberán ser atribuidas a la cuenta que corresponda al momento en que se generan. Ante diversas alternativas contables se optará por aquella que sea menos optimista, esto es el menos favorable para la empresa, a efectos de no sobreestimar los activos y utilidades.

La aplicación del principio de Prudencia se evidencia entre otros en la valuación de los valores negociables e inventarios, al adoptar la regla de "costo o mercado, el que sea más bajo"; así por ejemplo, si se cuenta con mercaderías que a los 30 días de su compra si incrementó el precio de adquisición en $20 \%$, no debe aumentar en libros el valor del inventario ni reconocer utilidades, porque estas no se han realizado, están todavía en poder de la empresa. Únicamente se reconocería la utilidad, en caso de su venta.

La aplicación del principio de Prudencia puede en ciertas circunstancias dejar de lado la aplicación de los principios de
Uniformidad o Valuación al Costo, adoptando el tratamiento de valuación a Valor de Mercado, como es el caso del rubro de Inventarios, en el supuesto que su valor al costo de adquisición, esté por encima del valor de mercado, debiéndose formular un ajuste contable por desvalorización de existencias.

Dante S. Basile (Argentina) en su obra: "Los Principios de Contabilidad Generalmente Aceptados" expone su posición sobre este principio:

"Las pérdidas deben ser atribuidas toda vez que existe la evidencia respaldatoria del factor que la produce, prueba respaldatoria que participa del principio de objetividad ...".

"Las ganancias cuando se verifiquen los hechos sustanciales que las generan, exista medición objetiva de ellas, y seguridad razonable de su materialización".

\section{UNIFORMIDAD}

Enunciado textual, según el PCGR:

Los principios generales, cuando fueren aplicables y las normas particulares - principios de valuación-utilizados para formular los estados financieros de un determinado ente deben ser aplicados uniformemente de un ejercicio a otro. Se señala por medio de una nota aclaratoria, el efecto en los estados financieros de cualquier cambio de importancia en la aplicación de los principios generales y de las normas particulares -principios de valuación-.

Sin embargo, el principio de la Uniformidad no debe conducir a mantener inalterables aquellos principios generales -principio de la uniformidad, cuando fuere aplicable, o normas particulares -principio de 
valuación-que las circunstancias aconsejen sean modificados.

\section{Comentario:}

Lo enunciado implica que las empresas una vez que hayan decidido la aplicación de una norma o método contable, todas las operaciones siguientes deberán ser tratadas en la misma forma, porque de lo contrario los cambios alterarían los rubros de los estados financieros, dificultando o haciendo impracticable la comparación de los rubros de un período a otro.

El principio de Uniformidad tiene relación directa con el concepto de Consistencia, incluso, algunos tratadistas lo consideran sinónimos; así Eric L. Kohler en su obra "Diccionario para Contadores", define a la consistencia en los siguientes términos: "Uniformidad constante durante un periodo, o de un periodo a otro, en los métodos de contabilidad, principalmente en las bases de valuación y en los métodos de acumulación reflejados en los estados financieros de una empresa comercial o de otra unidad contable o económica. Existen tres tipos generalmente reconocidos como consistencia; la "vertical", que se observa dentro de un grupo de estados financieros relacionados entre sí que llevan la misma fecha; la "horizontal", que se observa en los estados financieros de un período a otro; $y$ una clase de consistencia "tridimensional" en una fecha particular, en comparación con organizaciones del mismo tipo u otras organizaciones en general...".

El principio de uniformidad aplicado a la comparabilidad de los estados financieros, de un ejercicio a otro, puede ser afectado por distintas circunstancias, sean inherentes o ajenas a la contabilidad, como:

a) Cambios en los PCGA o en los métodos de aplicación de los mismos; como por ejemplo, cambio en el método de valuación de las existencias, de PEPS (primero que entra, primero que sale) a promedio ponderado.

b) Cambios en los criterios de medición de los activos o pasivos con incidencia en la situación financiera y en el resultado económico; como por ejemplo, cambio en el método de depreciación de los activos fijos, de línea recta a horas máquina; esto es de asumir gastos de depreciación anual por sumas iguales, cuyo total al término de la vida de servicio del activo fijo será igual al costo del mismo, pasar a asumir gastos de depreciación en una cuota fija por horas de uso de la máquina, la cual se determina estimando el número total de horas que el activo fijo estará en uso durante su vida de servicio.

c) Cambios que afectan al ejercicio, producto de la corrección de errores de ejercicio anteriores. No obstante del correcto proceder se ha generado inconsistencia.

d) Cambios en las condiciones productivas de la empresa que altera su rentabilidad, y como tal la comparabilidad de los saldos de las cuentas respecto a ejercicio precedentes; como por ejemplo, vender y no reemplazar parte de las máquinas y equipos de producción que reduce sustancialmente su capacidad de producción y entre otros sus ventas y gastos de un ejercicio a otro.

e) Cambios por hechos excepcionales o circunstanciales que inciden en el patrimonio esporádicamente; como por ejemplo exportación no asegurada de bienes perecibles, dañado durante el trayecto, que obviamente no será cobrado al cliente, y que necesariamente será asumido como pérdida.

La aplicación del principio de Uniformidad a los actos del registro contable, está supeditado a la clase de actividad o giro 
de la empresa, por lo que no siempre se ha de aplicar iguales tratamientos contables; por ejemplo, los préstamos obtenidos de un banco por una empresa industrial, para incrementar su capital de trabajo, ha de generar intereses, éstos serán contabilizados aplicando el principio de devengado, usualmente a gastos del periodo; pero si el préstamo ha sido obtenido por una empresa agraria para apoyar el financiamiento de su campaña agrícola, cuya cosecha ha de obtenerse en el siguiente ejercicio, los intereses serán diferidos a través de la cuenta 236 cultivos en proceso.

En caso se hubieran efectuado cambios importantes en los métodos de valuación y registro de un periodo a otro, deberá esto revelarse en una nota a los estados financieros en el año en que se efectuó el cambio, explicando la naturaleza de la diferencia del método, los motivos justificatorios del cambio y su efecto financiero y en los resultados, en comparación al resultado que se hubiera obtenido de haber continuado con el mismo método del año anterior; es decir, que es necesario cuantificar su efecto en los rubros más importantes de los estados financieros.

\section{SIGNIFICACIÓN O IMPORTANCIA RELATIVA}

Enunciado textual, según el PCGR:

Al ponderar la correcta aplicación de los principios generales y las normas particulares, es necesario actuar con sentido práctico. Frecuentemente se presentan situaciones que no encuadran dentro de aquellos y que, sin embargo, no presentan problemas porque el efecto que producen no distorsiona el cuadro general.

Desde luego, no existe una línea demarcatoria que fije los límites de lo que es y no es significativo, consecuentemente, se debe aplicar el mejor criterio para resolver lo que corresponde en cada caso, de acuerdo a las circunstancias, teniendo en cuenta factores tales como el efecto relativo en el activo, pasivo patrimonio o en el resultado de las operaciones.

\section{Comentario:}

El principio de Significación, también denominado Materialidad, está dirigido complementariamente a dos aspectos principales de la contabilidad:

a) Cuantificación o medición del patrimonio.

b) Exposición de partidas en los estados financieros.

La relación del principio de significación con la medición del patrimonio está referido a que debe haber flexibilidad para admitir mediciones que no respondan a lo prescrito por la disciplina contable (errores, violación de los principios, etc.), que incidan en los resultados del ejercicio, pese a lo cual, sin mediar los ajustes correspondientes, los estados financieros pueden ser admitidos como instrumentos informativos válidos de la situación patrimonial, financiera y económica del ente a que están referidos.

Las transacciones de menor cuantía, no serán necesariamente tratadas de acuerdo a los Principios de Contabilidad Generalmente Aceptados; se hacen excepciones en su aplicación, así por ejemplo, por los activos fijos adquiridos, en aplicación del principio de Realización, se afecta periódicamente su costo a resultados vía cuenta divisionaria 681 Depreciación de inmuebles, maquinaria y equipos, a partir del mes en que estos bienes sean utilizados en la generación de ingresos; pero si se hubiera comprado una calculadora electrónica pequeña a $\mathrm{S} / .50$, registrarlo como activo fijo, dado su larga 
vida útil, tendría mínima incidencia en el Balance General e implicaría adoptar medidas complementarias de control de activos fijos, por lo que bajo el principio de Significatividad, se cargaría en libros como gastos del periodo.

En el caso del registro de las existencias, es recomendable aplicar un sistema de Inventarios Permanentes, que implique ojercer un control físico y monetario a través de documentos de entradas y salidas, así como de registros auxiliares, pero puede suceder que ejercer un control permanente sobre estos, no sea importante para la empresa y que además implique su control un elevado costo operativo, entonces podría optarse por cambiar a un sistema de Inventarios Periódicos por todo o parte de las existencias, y que para efectos de asumir el gasto o costo del periodo por los artículos utilizados o consumidos, deberá practicarse un inventario físico a determinada fecha y que previa valorización de éstos y cálculos correspondientes, se procederá a ajustar el saldo de las cuentas de existencias a lo efectivamente encontrado. Este tratamiento no será rigurosamente correcto, pero si razonable y viable su aplicación bajo los alcances del principio de Significación.

El criterio general del principio de Significación es dar importancia a lo que razonablemente lo tiene desechando lo trivial. Ante la dificultad que reviste diferenciar lo que es importante de aquello que no lo es, el contador debe tener una apreciación objetiva de los hechos, siendo necesario que recurra a su buen criterio profesional y sentido práctico para evaluar los acontecimientos. En contraparte una transacción registrada es importante, si ésta al ser revelado a un usario de los estados financieros, influyera en sus decisiones sobre el ente.

\section{EXPOSICIÓN}

\section{Enunciado, según el PCGR:}

Los estados financieros deben contener toda la información y discriminación básica y adicional que sea indispensable para una adecuada interpretación de la situación financiera y de los resultados económicos del ente a que se refieren.

\section{Comentario:}

El principio de Exposición, también denominado Revelación Suficiente, implica formular los estados financieros en forma comprensible para los usuarios. Tiene relación directa con la presentación adecuada de los rubros contables que agrupan los saldos de las cuentas, para una correcta interpretación de los hechos registrados; así por ejemplo un anticipo concedido a un proveedor, no se debe mostrar restado del saldo del rubro Cuentas por Pagar Comerciales (pasivo), sino incluir en el saldo de Cuentas por Cobrar Comerciales (activo), evitando mostrar a menor suma las obligaciones comerciales, cuando más bien el efecto de la transacción es que incrementa las cuentas por cobrar, al haberse evidenciado derechos de cobro (recuperación) a efectivizar en el futuro.

Lo expuesto implica no ocultar hechos o cosas, incluso con posterioridad a la fecha de emisión de los estados financieros, ni exagerar los detalles informativos, a efectos de no distorsionar la interpretación y consecuente toma de decisiones dentro y fuera del ámbito de la empresa. Las notas que se acompañan a los estados financieros deben contener datos esenciales y adecuadamente redactados para la comprensión del usuario. Evitar aplicar un enfoque subjetivo en la interpretación de los datos a revelar. 
El principio de Exposición está íntimamente ligado al de Significatividad, el que se adopta como criterio para revelar hechos relevantes, por consiguiente se debe exponer sucesos como: Contingencias; compromisos relativos a la adquisición de bienes o servicios; convenios y condiciones contractuales referidos a préstamos obtenidos, amortizaciones, restricciones en la distribución de utilidades, gravámenes sobre bienes (hipotecas); información sobre el enfoque aplicado en la valuación de los recursos económicos, vigencia y cambio de estos valores en el tiempo; contratos importantes, sobre todo con empresas afiliadas u accionistas; descripción del giro o actividades de la empresa, si no se puede identificar de inmediato en los estados financieros.

\section{BIBLIOGRAFIA}

BASILE, Dante Sebastian

1977."Los principios de contabilidad generalmente aceptados". Ediciones Nueva Técnica S.R.L. Buenos Aires, Argentina.

RESOLUCIÓN CONASEV N ${ }^{\circ}$ 006-84- EFC/ 94.10 del 15/02/84.

Plan Contable General Revisado.

SOLOMON, Lanny /VARGO, Richard/SCHOROEDER, Richard

1988. "Principios de contabilidad", HARLA

S.A. de C.V. Edición, México.

KOHLER, L.Eric

1974."Diccionario para contadores".

México. 